\title{
Electronic structure analysis of quasi-one-dimensional monophosphate tungsten bronzes
}

\author{
A. Mascaraque, ${ }^{1}$ L. Roca,,${ }^{1,2}$ J. Avila, ${ }^{1,2}$ S. Drouard, ${ }^{3}$ H. Guyot, ${ }^{3}$ and M. C. Asensio ${ }^{1,2}$ \\ ${ }^{1}$ LURE, Centre Universitaire Paris-Sud, Bât. 209D, B.P. 34, 91405 Orsay Cedex, France \\ ${ }^{2}$ Instituto de Ciencia de Materiales, CSIC, 28049 Madrid, Spain \\ ${ }^{3}$ LEPES-CENES, B.P. 166x, 38042 Grenoble, Cedex 9, France
}

(Received 26 November 2001; revised manuscript received 5 March 2002; published 4 September 2002)

\begin{abstract}
Synchrotron-radiation angle-resolved photoemission measurements were performed in the nondoped $\left(\mathrm{PO}_{2}\right)_{4}\left(\mathrm{WO}_{3}\right)_{4}\left(\mathrm{WO}_{3}\right)_{4}$ compound in order to obtain a detailed picture of the electronic structure. This material is a low-dimensional compound due to the strong anisotropic structure built up with chains. The measurements were done at room temperature, probing different crystalline directions. Three states are observed in the vicinity of the Fermi level: one of them exhibits one-dimensional character while the other two are of twodimensional nature. A very good agreement has been found between these electronic bands and theoretical calculations based on the hidden nesting mechanism.
\end{abstract}

DOI: 10.1103/PhysRevB.66.115104

PACS number(s): 79.60.-i, 71.45.Lr, 64.60.-i, 73.20.At

\section{INTRODUCTION}

The physical properties of both organic and inorganic quasi-low-dimensional solids have been the subject of numerous investigations, since these materials are excellent models to test fundamental theories of low-dimensional systems. ${ }^{1}$ In recent years, several studies have been focused on the study of the chemical and physical properties of families of transition metal oxides, chacolgenides, and bronzes. The main reason for such studies are the quasi-one- or quasitwo-dimensional properties that these compounds present. ${ }^{1,2}$ Their strong structural anisotropy is reflected in a variety of electronic properties and other interesting characteristics, including quasi-low-dimensional transport, anomalous magnetic properties, metal-insulator and metal-metal transitions, periodic lattice distortions, and charge-density-wave (CDW) phase transitions.

In general, low-dimensional metallic oxides exhibit two kinds of electronic instabilities: a CDW or a superconducting state. In both cases the instability leads to a gap. However, in spite of the large amount of work devoted to these materials, the mechanism that determines why one or another transition takes place is not yet understood.

The CDW phases observed in molybdenum and tungsten bronzes have been explained within the context of the hidden nesting model. ${ }^{3}$ A CDW transition takes place when a lowdimensional metal reduces its electronic energy by opening a gap as a consequence of a structural rearrangement. This situation is energetically favorable only in low-dimensional systems. In this case it is easier to find large portions of the metal Fermi surface that exhibit parallel areas which present nesting. In this scenario the electron-phonon coupling is strong enough to stabilize a CDW. In the hidden nesting model, the Fermi surface of the metallic phase of a lowdimensional compound can be decomposed in different onedimensional (1D) Fermi surface sheets, formed by parallel lines that exhibit a large nesting. ${ }^{3}$

Phosphate tungsten bronzes are a family of metallic bronzes with quasi-low-dimensional structural and electronic properties similar to those observed in the molybdenum bronzes. ${ }^{4,5}$ The monophosphate tungsten bronzes (MPTB) with pentagonal tunnels are a family of low-dimensional conductors with a general formula $\left(\mathrm{PO}_{2}\right)_{4}\left(\mathrm{WO}_{3}\right)_{p}\left(\mathrm{WO}_{3}\right)_{q}$. As in the molybdenum bronzes, the essential building blocks are perovskite-type layers made up of $\mathrm{WO}_{6}$ octahedra and linked across $\mathrm{PO}_{4}$ tetrahedra. ${ }^{1}$ In the junction between two $\mathrm{W}-\mathrm{O}$ layers, pentagonal tunnels are formed. The $p$ and $q$ indices indicate the number of $\mathrm{WO}_{6}$ octahedra per unit cell needed to form the $\mathrm{W}-\mathrm{O}$ layers. In our particular case, $p$ $=q=m$ and then the general formula is reduced to $\left(\mathrm{PO}_{2}\right)_{4}\left(\mathrm{WO}_{3}\right)_{2 m}$. Due to the layer structure, all the tungsten bronzes present a $2 \mathrm{D}$ character. ${ }^{6-8}$ However, some of them have an even more reduced dimensionality (1D). ${ }^{1}$

The electronic behavior of the members of the MPTB family is very rich. For compounds with $m=4,6,7$ two consecutive CDW's have been observed. ${ }^{9}$ However, there are strong differences in the electronic and magnetic properties as a function of $m$. In addition to this, the $m=2$ member is semiconducting and presents antiferromagnetic order. ${ }^{10}$

Most of the experimental studies performed on molybdenum and tungsten bronzes have focused either on the analysis of the geometrical structure or on the examination of the macroscopical electric and magnetic properties. However, in spite of its crucial importance, the electronic structure has been much less studied. In this paper, we present a detailed determination of the electronic structure of nondoped tungsten bronzes. We examine the electronic structure of the $\left(\mathrm{PO}_{2}\right)_{4}\left(\mathrm{WO}_{3}\right)_{2 m}$ compound with $m=4$. We analyze the band dispersion along the three high-symmetry directions $(\overline{\Gamma X}$, $\overline{\Gamma Y}$, and $\overline{\Gamma M}$ ) using detection geometries sensitive to different symmetries, in order to differentiate the electronic states and to obtain a deeper understanding on the electronic behavior. The experimental data are compared with electronic theoretical calculations which proposed that the CDW transition is due to a hidden nesting mechanism.

\section{GEOMETRIC STRUCTURE}

The molybdenum and tungsten bronzes have been synthesized and their structure has been studied since the early 1980s. All the family crystallizes in an orthorhombic structure that is built up from perovskite prototype $\mathrm{ReO}_{3}$ - 


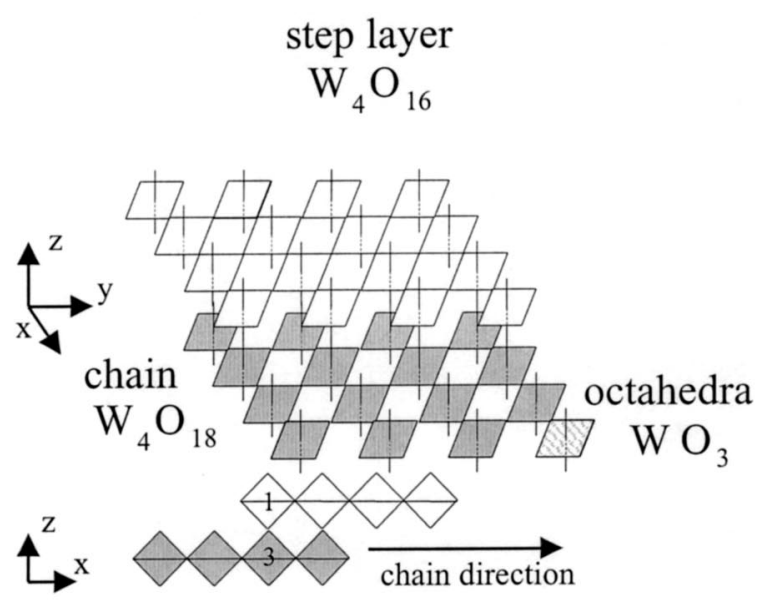

a)

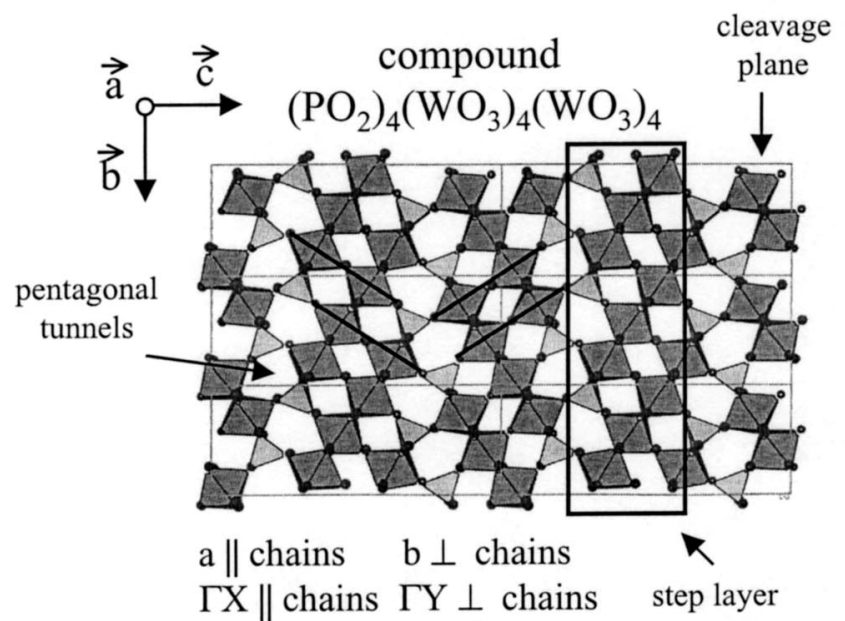

b)

FIG. 1. Geometric structure of the $\left(\mathrm{PO}_{2}\right)_{4}\left(\mathrm{WO}_{3}\right)_{4}\left(\mathrm{WO}_{3}\right)_{4}$ compound. Panel (a) summarizes the formation process of a step layer: several $\mathrm{WO}_{3}$ octahedra linked together to give rise to $\mathrm{W}_{4} \mathrm{O}_{18}$ chains; then, two chains joined with a $(1,3)$ condensation to produce a $\mathrm{W}_{4} \mathrm{O}_{16}$ step layer. Panel (b) represents the final structure of the material. See text for details.

type infinite layers of $\mathrm{WO}_{3}$ octahedra separated by $\mathrm{PO}_{4}$ tetrahedra.

One of the most important characteristics of these materials is their structural flexibility, which is an advantage for the analysis of the electronic interactions. The strong anisotropic layered structure makes them ideal systems to study the effects of electron confinement in the macroscopic properties.

A $\left(\mathrm{PO}_{2}\right)_{4}\left(\mathrm{WO}_{3}\right)_{2 m}$ compound is fully described by the value of $m$, which determines the width of the step layers $(m / 2)$ and the pair $(r, s)$, which describes how adjacent chains are condensed. The $(r, s)$ indices mean that the $r$ th octahedron of the first chain shares the oxygen axial atom with the $s$ th octahedron of the second chain. In these materials the crystallographic values $\vec{a}$ and $\vec{b}$ are mostly independent of $m$, while $\vec{c}$ is directly related with the thickness of the $\mathrm{W}-\mathrm{O}$ step layers.
In our particular case, for the $\left(\mathrm{PO}_{2}\right)_{4}\left(\mathrm{WO}_{3}\right)_{4}\left(\mathrm{WO}_{3}\right)_{4}$ compound, four $\mathrm{WO}_{3}$ octahedra are linked together forming a $\mathrm{W}_{4} \mathrm{O}_{21}$ unit. These units are joined to form a $\mathrm{W}_{4} \mathrm{O}_{18}$ chain along the $y$ direction and they run along $\vec{a}$ and $\vec{a} \pm \vec{b}$ crystallographic directions, as shown in Fig. 1(a). In the next step, two chains condense to produce a $\mathrm{W}_{4} \mathrm{O}_{16}$ step layer with $(1,3)$ condensation. Finally, these step layers are linked to each other across an infinite plane of $\mathrm{PO}_{4}$ tetrahedra, forming layers along the $\overrightarrow{a b}$ plane of the unit cell. This junction creates pentagonal tunnels, formed by the corner sharing of three $\mathrm{WO}_{3}$ octahedra and two $\mathrm{PO}_{4}$ tetrahedra [Fig. 1(b)]. There is an inclination between neighboring step layers. Thus following layers run forming a zigzag. The whole formation process is schematized in Fig. 1. The orthorhombic unit cell parameters are $\vec{a}=5.285 \AA, \vec{b}=6.569 \AA$, and $\vec{c}$ $=17.351 \AA$, and the compound crystallizes in the $P 2{ }_{1} 2{ }_{1}{ }_{1}$ space symmetry group. ${ }^{11}$ It is important to stress the existence of two kinds of $\mathrm{W}$ atoms sites, depending which corners of the $\mathrm{WO}_{3}$ octahedra they share. Some $\mathrm{WO}_{3}$ octahedra share $\mathrm{O}$ atoms only with other $\mathrm{WO}_{3}$ octahedra, while a second kind share $\mathrm{O}$ atoms with $\mathrm{PO}_{4}$ tetrahedra also. These different environments have important implications in the electronic behavior.

\section{EXPERIMENT}

The experiments were carried out in the Spanish-French beamline at LURE (Orsay), receiving synchrotron radiation from SU8 insertion device of SuperAco storage ring. All the data were taken at room temperature, above the CDW phase transition temperature.

The samples were grown following the chemical vapor transport technique. ${ }^{7}$ The typical size was $2 \times 2 \mathrm{~mm}^{2}$ of a platelike purple crystal. The samples were prepared in situ by cleaving the crystals in ultrahigh vacuum. The chamber base pressure was in the $10^{-11}$ mbar range. Typical energy and angular resolution were $50 \mathrm{meV}$ and $0.5^{\circ}$, respectively. In order to avoid electron damage to the sample, ${ }^{12}$ the orientation was done in two steps. Before the experimental measurements, the sample was aligned using forward-scattering photoelectron diffraction, in order to determine the $\overline{\Gamma X}$ and $\overline{\Gamma Y}$ high-symmetry directions. Later on and after the data were acquired, this orientation was confirmed by low-energy electron diffraction (LEED). The quality of the sample was monitored during the data acquisition by measuring the spectral intensity between the conduction and valence bands.

\section{ELECTRONIC STRUCTURE}

The transport properties of Mo blue bronzes are extremely anisotropic. ${ }^{1}$ At room temperature, the resistivity along the $\vec{b}$ axis is at least one or two orders of magnitude larger than along the other two axes. ${ }^{1,2}$ This behavior is common for all families of bronzes, and it is easily understood in view of the electronic structure.

The electronic properties of $\mathrm{WO}_{3}$ were first explained by Goodenough ${ }^{13}$ who described the electronic structure within a simple model. Each $\mathrm{O}$ atom is $s p$ hybridized. The central $\mathrm{W}$ 


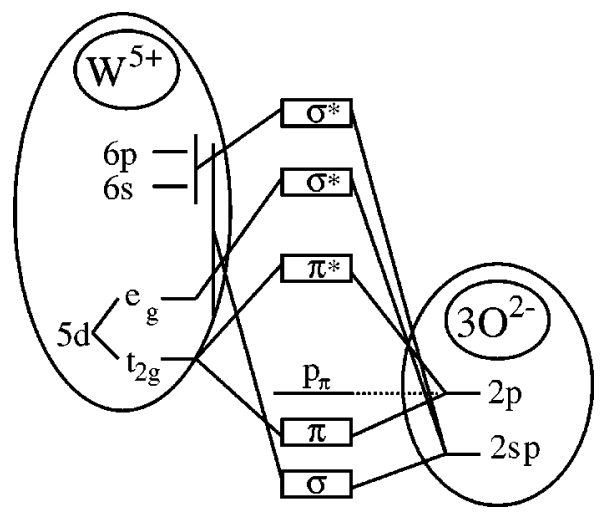

FIG. 2. Schematic diagram of the energetic levels involved in the electronic structure (Ref. 15).

atom of the octahedron has $6 s, 6 p$, and $5 d\left(e_{g}\right)$ orbitals which overlap with the O sp hybrids to form $\sigma$ and $\sigma^{*}$ bands. In the same way, the $\mathrm{W} 5 d\left(t_{2 g}\right)$ orbitals overlap with the $\mathrm{O} p$ to form $\pi$ and $\pi^{*}$ bands (see Fig. 2). In MPTB, the $t_{2 g}$ bands are partially filled by electrons coming from the $\mathrm{PO}_{4}$ groups, accounting for the metallic character of the compound. The dispersive properties of the $d$-block bands can be deduced by determining whether the bridging $\mathrm{O} p$ orbitals can mix with the metal $d$ orbitals or not.

More accurate theoretical tight-binding band calculations performed by Canadell et al. ${ }^{8,14,15}$ based upon the extended Hückel method have shown that the electronic structure is very similar for all compounds of the MPTB family. Their essential features give rise in the calculation to the ideal $\mathrm{W}_{4} \mathrm{O}_{16}$ step layer, common in all families. Due to the layered character of MPTB compounds, the surface projection of the Brillouin zone is straightforward: the rectangle formed by $\Gamma, X, Y$, and $S$ bulk symmetry points becomes the surface Brillouin zone formed by $\bar{\Gamma}, \bar{X}, \bar{Y}$, and $\bar{M}$ symmetry points, respectively.

The results of the calculation are shown in Fig. $3,{ }^{8,15}$ which represents the bottom part of the electronic band structure of the $t_{2 g}$ bands calculated for a real

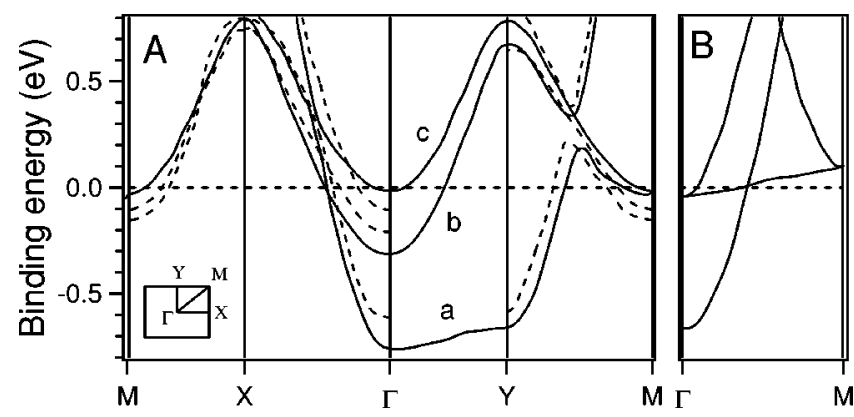

FIG. 3. Solid lines: theoretical calculations performed using the extended Hückel method for a real $\left(\mathrm{PO}_{2}\right)_{4}\left(\mathrm{WO}_{3}\right)_{4}\left(\mathrm{WO}_{3}\right)_{4}$ compound (panel A) and for an ideal $\mathrm{W}_{4} \mathrm{O}_{16}$ step layer (panel $\mathrm{B}$ ). The theoretical bands have been multiplied by a factor (see text for details). Dashed lines: ab initio density functional theoretical calculations performed along some relevant surface directions for the $\left(\mathrm{PO}_{2}\right)_{4}\left(\mathrm{WO}_{3}\right)_{4}\left(\mathrm{WO}_{3}\right)_{4}$ compound. The inset schematizes the surface Brillouin zone.
$\left(\mathrm{PO}_{2}\right)_{4}\left(\mathrm{WO}_{3}\right)_{4}\left(\mathrm{WO}_{3}\right)_{4}$ compound (panel A) and an ideal $\mathrm{W}_{4} \mathrm{O}_{16}$ step layer (panel B). ${ }^{16}$ Three bands appear in the region near the Fermi level (the top of the conduction band), labeled $\mathbf{a}, \mathbf{b}$, and $\mathbf{c}$. Band a has quasi-1D character. It is flat along the $\overline{\Gamma Y}$ direction and disperses in other high-symmetry directions. It crosses the Fermi level along $\overline{\Gamma X}, \overline{Y M}$, and $\overline{\Gamma M}$. The 1D character of band $\mathbf{a}$ is due to a very weak interaction between adjacent chains ( $\overline{\Gamma Y}$ direction or $\vec{b}$ axis $)$ and strong conduction along the chains $(\overline{\Gamma X}$ direction or $\vec{a}$ axis).

Bands $\mathbf{b}$ and $\mathbf{c}$ have both 2D character. They come from the splitting of a doubly degenerate band obtained in the calculations of the ideal W-O layer. During the formation of the $\mathrm{W}_{4} \mathrm{O}_{18}$ chain, the tungsten octahedra suffer a local distortion to reduce the strain. This distortion is mainly a rotation around the vertical axis. When the calculations are performed in a real distorted $\mathrm{WO}_{3}$ octahedron, the double degeneration of the upper $2 \mathrm{D}$ band is lifted. The upper two bands present a strong dispersion due to the interaction between the $\mathrm{W}_{4} \mathrm{O}_{18}$ chains. They have lower binding energy than band a and cross the Fermi level along all highsymmetry directions.

The theoretical bands calculated by the extended Hückel method have been multiplied by a factor in order to reproduce the real value of the binding energy (BE) of the experimental data. A factor of 3.75 was chosen so that the BE of the flat band a along the $\overline{\Gamma Y}$ direction was reproduced by the theoretical calculations. The same factor was used for all symmetry directions.

$A b$ initio density functional theory calculations have been performed more recently for the $m=4$ member. ${ }^{17}$ The theoretical results are similar to those obtained by Canadell et al. The results of the calculation performed by Sandre et al. are represented as dashed lines in Fig. 3. The ab initio electronic structure obtained has the same shape as that calculated by the Hückel method. However, the $a b$ initio bandwidth is much closer to the experimental one, and no multiplication factor is needed in order to reproduce the experimental data.

As discussed above, the electronic properties of these layered compounds are governed by the bottom three $t_{2 g}$ bands, which are partially filled. Since the electronic structure is almost identical for $\left(\mathrm{PO}_{2}\right)_{4}\left(\mathrm{WO}_{3}\right)_{4}\left(\mathrm{WO}_{3}\right)_{4}$ and for $\mathrm{W}_{4} \mathrm{O}_{16}$ (and very similar to $\mathrm{Mo}_{4} \mathrm{O}_{16}$ ), the orbital character of the three bands can be easily deduced from the calculations performed for the $\mathrm{Mo}_{4} \mathrm{O}_{16}$ layer. In this compound and at the $\Gamma$ point, band a has $d_{\left(x^{2}-y^{2}\right)}(\delta$ character $)$, while bands $\mathbf{b}$ and $\mathbf{c}$ have $d_{x y}$ and $d_{y z}$ character, respectively. Using synchrotron radiation and an adequate experimental setup, it is possible to exploit the symmetry of the wave functions in order to discriminate between the three different bands. To this end, the plane defined by the light and emitted electron (detection plane) must be a mirror plane of the crystal, ${ }^{18}$ and so all the symmetry relationships are related to this plane. Within the dipolar approximation, the photoemission signal $I$ is proportional to the matrix element governing the photoemission process,

$$
I \sim\left\langle\psi_{f}|\vec{A} \nabla| \psi_{i}\right\rangle,
$$



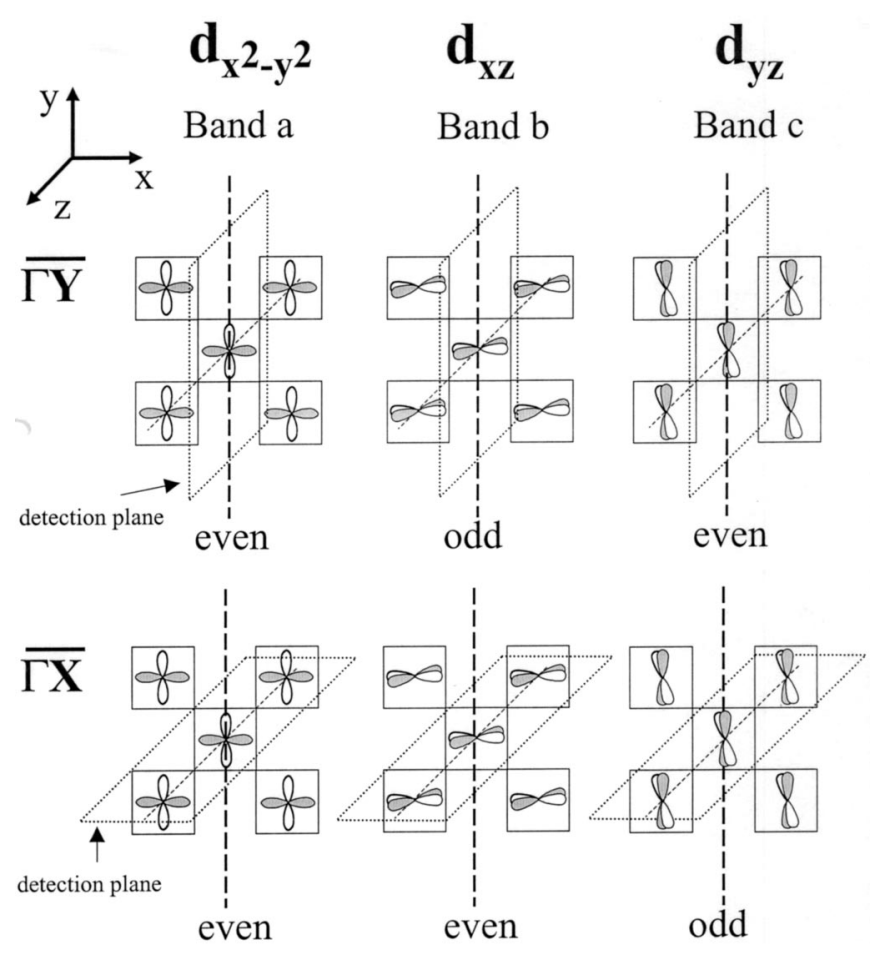

FIG. 4. Schematic representation of the symmetry of the wave functions with respect to the detection plane. See text for details.

where $\psi_{f}$ is the final-state wave function, $\psi_{i}$ the initial-state wave function, and $\vec{A} \nabla$ the momentum operator. In order to measure any photoemission signal in the analyzer, the final wave function should have even symmetry. When the dipolar vector of the radiation $\vec{A}$ lies inside the detection plane, the momentum operator is even with respect to this plane, and only even initial wave functions are detected. On the contrary, when $\vec{A}$ is perpendicular to the detection plane, $\vec{A} \nabla$ is odd, and the signal detected comes only from odd initial states. ${ }^{19}$

Due to the relatively complicated structure of these compounds, there is only one mirror plane: the plane perpendicular to the surface that contains the $\overline{\Gamma Y}$ direction. This means that the symmetry detection rules are strictly kept only along the $\overline{\Gamma Y}$ direction. However, the projection of the bulk on the surface has a mirror plane in the plane perpendicular to the surface which contains the $\overline{\Gamma X}$ direction (pseudomirror plane). Thus, although a complete extinction of the signal cannot be expected along $\overline{\Gamma X}$, it is reasonable to predict a photoemission intensity reduction of the orbitals with nonadequate symmetry along this plane. Applying the symmetry rules is then straightforward: along the $\overline{\Gamma Y}$ direction, the wave functions of a and $\mathbf{c}$ states have even symmetry with respect to the detection plane, so they should be detected in even symmetry. Band $\mathbf{b}$ has odd symmetry and should be measured in odd detection. On the contrary, along the $\overline{\Gamma X}$ direction, the wave functions of $\mathbf{a}$ and $\mathbf{b}$ states have even symmetry (odd symmetry for band c). Along the $\overline{\Gamma M}$ direction there is no mirror plane, so all bands should be experimentally detected. This is represented in Fig. 4.

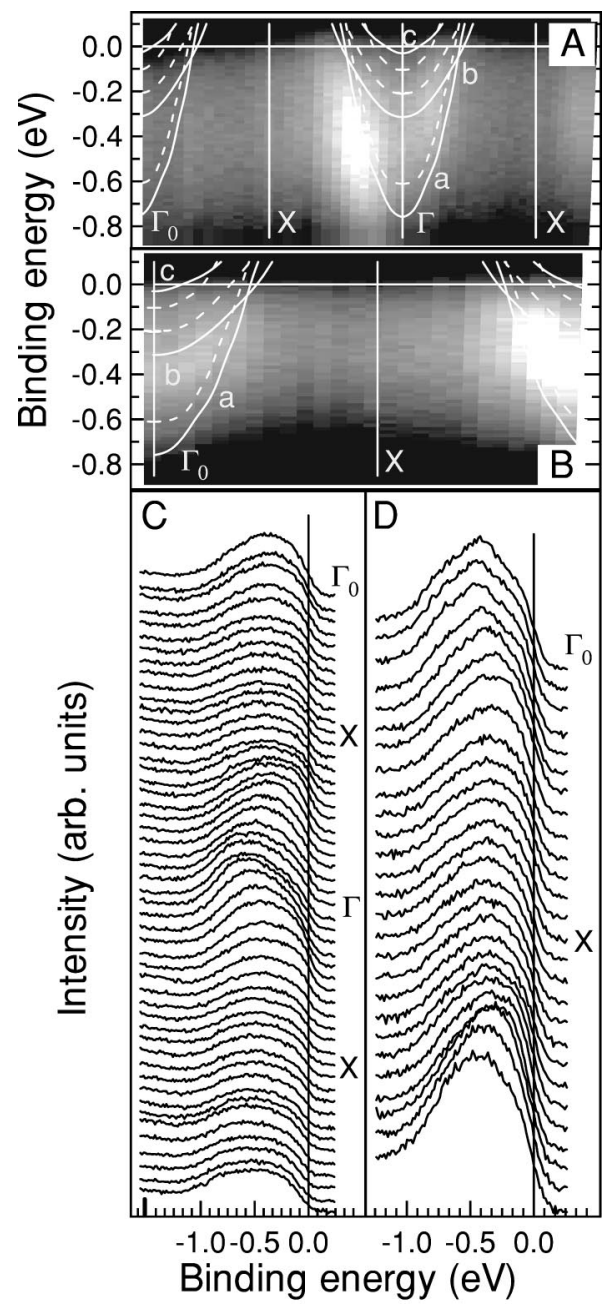

FIG. 5. Panels C and D: experimental EDC's obtained for the nondoped compound along the $\overline{\Gamma X}$ high-symmetry direction, for even (panel C) and odd (panel D) symmetry detection geometries. Panels A and B: the corresponding two-dimensional plots of the bottom EDC's with intensity represented as a gray scale. Photon energy was $h \nu=30 \mathrm{eV}$. Solid lines correspond to theoretical calculations using the extended Hückel method, while dashed lines are obtained from $a b$ initio theoretical calculations.

The number of $5 d$ electrons per W-O layer to fill up the bands is always 2, regardless the member of the MPTB family, i.e., the value of $m$. Nearly all MPTP compounds have two W-O layers per unit cell, so there exist four electrons per unit cell. However, the electron density per $\mathrm{W}$ ion $(\mathrm{m} / 2)$ depends on the particular compound geometry. This dependence of the electronic density makes it possible to change the electronic properties by just changing $m$. Because macroscopic properties are under more fundamental characteristics, as the electron-electron or electron-phonon interactions, these materials are a model system to study dimensionality effects without changing the electron counting.

The experimental electronic structure data for the $\left(\mathrm{PO}_{2}\right)_{4}\left(\mathrm{WO}_{3}\right)_{4}\left(\mathrm{WO}_{3}\right)_{4}$ compound is represented in Figs. 5 and 6 . The two lower panels show the experimental energy distribution curves (EDC's) as a function of emission angle. The upper panels show the EDC intensity data as a gray scale. 


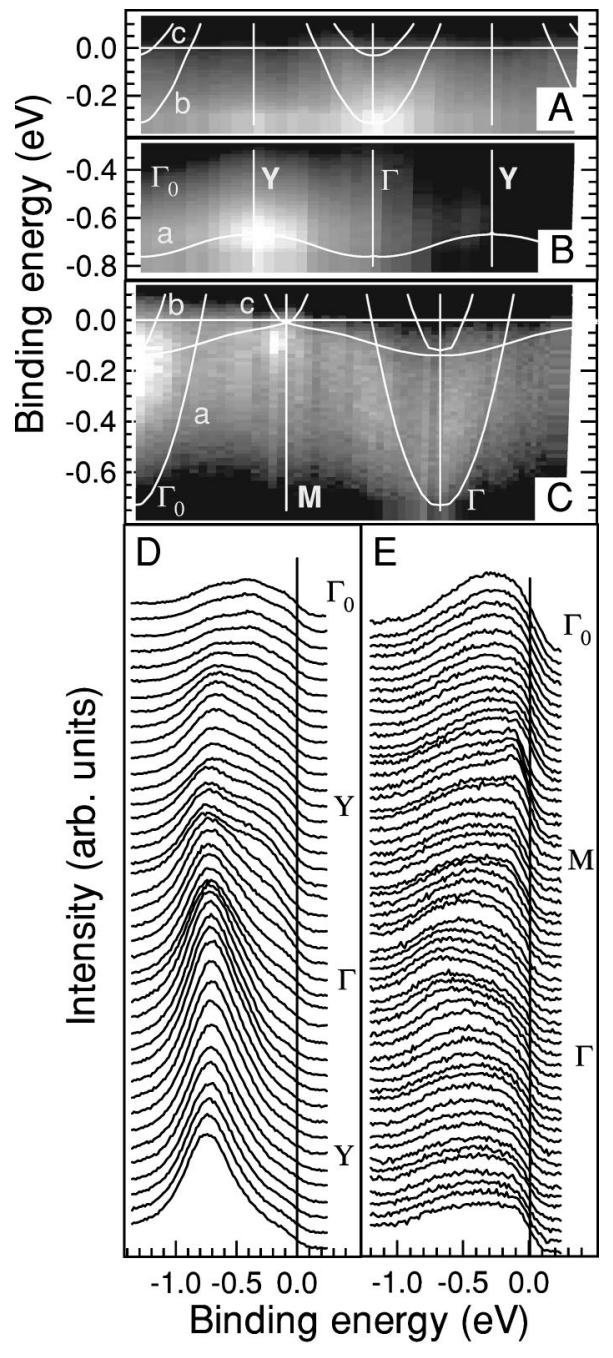

FIG. 6. Panels D and E: experimental EDC's obtained for the nondoped compound along $\overline{\Gamma Y}$ (panel D) and $\overline{\Gamma M}$ (panel E) highsymmetry directions, both taken for even detection geometry. Panels $\mathrm{A}, \mathrm{B}$, and $\mathrm{C}$ : the corresponding two-dimensional plots of the bottom EDC direction with intensity represented as a gray scale. In order to be able to observe the area near the Fermi level, two panels are plotted for the $\overline{\Gamma Y}$ direction. Photon energy was $h \nu=30 \mathrm{eV}$. Solid lines correspond to theoretical calculations performed by the extended Hückel method.

An important feature obtained from the EDC experimental data is the broadening of the states along the three highsymmetry directions. The origin of the broadening lies in the existence of two different kinds of $\mathrm{WO}_{3}$ octahedra, which are displaced from the ideal positions due to the distortion. ${ }^{20}$ This distortion generates different environments for the $\mathrm{W}$ and $\mathrm{O}$ atoms, which naturally enhances the spectral broadening. The spectral intensity between the valence and conduction bands reflects directly the defect concentration; thus, the intensity depletion measured in this region discards the possibility that the broadening is due to defects. This fact supports an intrinsic origin of the experimental broadening.

The broadbands make difficult the differentiation between the different half-filled states. Due to this reason, the experimental data have been measured both in even and odd sym- metry detection geometries with the aim of detecting and discriminating the three $\mathbf{a}, \mathbf{b}$ and $\mathbf{c}$ bands as a function of their symmetry. In Fig. 5 we represent the data along the $\overline{\Gamma X}$ high-symmetry direction in even- and odd-symmetry detections. The broadening is especially large at even detection. In this symmetry, a peak of $\sim 1.0 \mathrm{eV}$ width disperses from a BE of $-0.7 \mathrm{eV}$ at $\bar{\Gamma}$ points up to the Fermi level. The band crossing is reflected in the reduction of the photoemission intensity at the Fermi level and in the spectral density detected after the band crossing. A careful inspection reveals that more than one state exists inside the main peak. Two states are clearly observed: one state disperses at higher BE, while a second one is located near the Fermi level.

For odd geometry detection the three states are better resolved: there is a main peak centered at $-0.4 \mathrm{eV} \mathrm{BE}$, surrounded by two smaller peaks at higher and lower binding energy, respectively. The central peak has a strong dispersion, with minima at $\bar{\Gamma}$ points.

Figure 6 shows the EDC's for the $\overline{\Gamma Y}$ and $\overline{\Gamma M}$ directions, both taken in even symmetry. Along the $\overline{\Gamma Y}$ direction the peaks are more easily observed than along $\overline{\Gamma X}$, because of the smaller broadening $(\sim 0.3 \mathrm{eV})$. In this case, the photoelectron emission plane is a real mirror plane and the extinction of the bands with nonadequate symmetry is complete. However, along the $\overline{\Gamma M}$ direction, the broadening is much larger than along $\overline{\Gamma X}$ or $\overline{\Gamma Y}$ due to the lack of mirror plane.

Along the $\overline{\Gamma Y}$ direction, two states are observed in even detection. The most intense is a flat band, centered at $-0.7 \mathrm{eV}$ BE. The dispersion of the band is negligible $(0.1$ $\mathrm{eV}$ ). In addition to this intense peak, another weak peak is observed in the region near the Fermi level. This weak peak disperses from a minimum of binding energy at the $\bar{\Gamma}$ points $(-0.3 \mathrm{eV})$ and crosses the Fermi level halfway between the $\bar{\Gamma}$ and $\bar{Y}$ points (see Fig. 6, panel A).

Along $\overline{\Gamma M}$ all three bands are detected and they exhibit a clear dispersion. The lower band disperses from $-0.8 \mathrm{eV} \mathrm{BE}$ at $\bar{\Gamma}$ up to the Fermi level. A second one has small dispersion, but approaches the Fermi level near the $\bar{M}$ point. A third one is detected as an increase of the intensity near the Fermi level around the $\bar{\Gamma}$ point. In this high-symmetry direction, the agreement between experiment and theoretical calculations is worse than in other directions. This is because the calculations are performed for an ideal $\mathrm{W}_{4} \mathrm{O}_{16}$ step layer. In the $\bar{\Gamma}$ point the experimental bands agree better with the theoretical bandwidths obtained for the real compound.

\section{DISCUSSION}

Our experimental results are fully consistent with the theoretical band structure determined for the $\left(\mathrm{PO}_{2}\right)_{4}\left(\mathrm{WO}_{3}\right)_{4}\left(\mathrm{WO}_{3}\right)_{4}$ compound, ${ }^{8,15,17}$ which indicates the presence of three states near the Fermi level: one with 1D character and two with $2 \mathrm{D}$ character.

In the upper panels of Figs. 5 and 6 we show gray-scale pictures of the experimental data and the theoretical bands from Fig. 3. In this kind of figure the comparison between data and predictions is very simple. As can be observed, the 
agreement between experiments and calculations is excellent. Along the $\overline{\Gamma X}$ direction, in even detection, one broad and dispersive peak is observed. This peak corresponds to bands $\mathbf{a}$ and $\mathbf{b}$. Band $\mathbf{b}$ is detected as an increase of the intensity near the Fermi level around the $\bar{\Gamma}$ points. On the contrary, band $\mathbf{c}$ is observed in odd detection, with higher BE than $\mathbf{a}$ and $\mathbf{b}$. Along this direction, all states disperse. Since this direction is parallel to the chains, the dispersion indicates a strong degree of delocalization along the chains and explains the differences in the electronic transport between different axis.

Along the $\overline{\Gamma Y}$ direction the symmetry rules are fulfilled. A flat state detected corresponds with band $\mathbf{a}$. Its nondispersive character is an indication of the strong 1D nature of this state, supporting confinement along the $\overline{\Gamma Y}$ direction and the lack of interchain interaction. In addition to this state, an extra very weak peak is detected closer to the Fermi level. Its dispersion and $\mathrm{BE}$ agree well with the ones expected for band $\mathbf{c}$ which has $2 \mathrm{D}$ character. Band $\mathbf{b}$ is not detected because it is symmetry forbidden.

The theoretical band structure is also reproduced along $\overline{\Gamma M}$. The deeper peak corresponds to band $\mathbf{a}$, while band $\mathbf{b}$ is observed as a state near the Fermi level. Bands $\mathbf{b}$ and $\mathbf{c}$ are detected as an intensity increase around the $\bar{M}$ points.

The excellent agreement between the experimental data and theory along the three high-symmetry points towards the hidden nesting mechanism as being responsible for the CDW's being stabilized in these compounds.

\section{CONCLUSIONS}

We have investigated the electronic structure of the lowdimensional inorganic $\left(\mathrm{PO}_{2}\right)_{4}\left(\mathrm{WO}_{3}\right)_{4}\left(\mathrm{WO}_{3}\right)_{4}$ compound using different symmetry detection geometries. Three broadbands are observed: one band with $1 \mathrm{D}$ character along $\overline{\Gamma Y}$ and two 2D bands. These structures reveal electron confinement along the chains with negligible interchain electronic interaction. The comparison between experimental data and theoretical calculations performed by the extended Hückel method and $a b$ initio density functional theory is excellent. Although this leads us to think that hidden nesting is the mechanism behind the CDW phase observed at low temperature, further measurements of the Fermi surface in these compounds are planned in order to fully confirm it. ${ }^{21}$

\section{ACKNOWLEDGMENTS}

This work has been partially financed by the Spanish agency MCYT, under Grant No. MAT2002-03431. A.M. and L.R. thank Ministerio de Ciencia y Tecnología (Grant Nos. PF 0051683701 and ES 99 50858888, respectively) for financial support.
${ }^{1}$ Physics and Chemistry of Low Dimensional Inorganic Conductor, edited by C. Schlenker, J. Dumas, M. Greenblatt, and S. Van Smaalen, Vol. 354 of NATO Advanced Study Institute, Series B: Physics (Plenum, New York, 1996).

${ }^{2}$ Oxides Bronzes, edited by M. Greenblatt [Int. J. Mod. Phys. B 7, 3937 (1993)].

${ }^{3}$ M.-H. Whangbo et al., Science 252, 96 (1991).

${ }^{4}$ M. Greenblatt, Chem. Rev. 88, 31 (1988).

${ }^{5}$ M. Greenblatt, Acc. Chem. Res. 29, 219 (1996).

${ }^{6}$ E. Wang et al., Phys. Rev. B 39, 12969 (1989).

${ }^{7}$ Z.S. Teweldemedhin et al., Phys. Rev. B 46, 7897 (1992).

${ }^{8}$ E. Canadell and M.-H. Whangbo, Phys. Rev. B 43, 1894 (1991).

${ }^{9}$ J. Dumas et al., J. Solid State Chem. 147, 320 (1999).

${ }^{10}$ Z.S. Teweldemedhin et al., Solid State Chem. 95, 21 (1991).

${ }^{11}$ J.P. Giroult et al., Acta Crystallogr., Sect. B: Struct. Crystallogr. Cryst. Chem. 37, 2139 (1981).
${ }^{12}$ K.E. Smith, Annu. Rep. Prog. Chem., Sect. C: Phys. Chem. 90, 115 (1995).

${ }^{13}$ J.B. Goodenough, Bull. Soc. Chim. Fr. 1915, 1200 (1965); Czech. J. Phys., Sect. B 17, 304 (1967).

${ }^{14}$ E. Canadell et al., Inorg. Chem. 28, 1466 (1989).

${ }^{15}$ E. Canadell et al., Inorg. Chem. 29, 3871 (1990).

${ }^{16}$ Notice than the two different calculations give different values for the bandwidths at $\Gamma$ point. As can be expected, the comparison between experiment and theory favors the results obtained for the real $\left(\mathrm{PO}_{2}\right)_{4}\left(\mathrm{WO}_{3}\right)_{4}\left(\mathrm{WO}_{3}\right)_{4}$ compound.

${ }^{17}$ E. Sandre et al., Phys. Rev. Lett. 86, 5100 (2001).

${ }^{18}$ F.J. Himpsel, Adv. Phys. 32, 1 (1983).

${ }^{19}$ J. Hermanson, Solid State Commun. 22, 9 (1977).

${ }^{20}$ J. Ludecke et al., Europhys. Lett. 49, 357 (2000).

${ }^{21}$ L. Roca et al. (unpublished). 